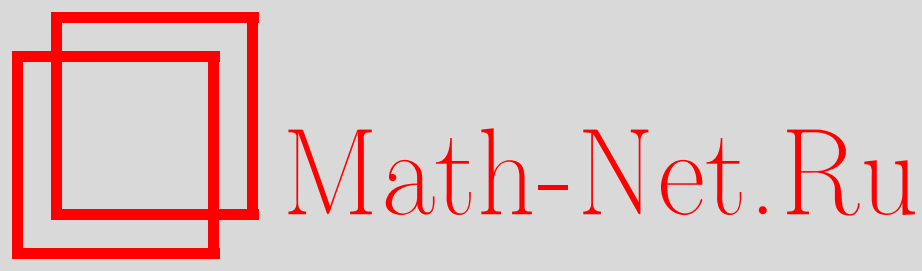

А. П. Сайко, О применении усреднения КрыловаБоголюбова-Митропольского к построению эффективных гамильтонианов в теории сильно коррелированных электронных систем, ТМФ, 2009, том 161, номер 2, 287294

DOI: https://doi.org/10.4213/tmf6439

Использование Общероссийского математического портала Math-Net.Ru подразумевает, что вы прочитали и согласны с пользовательским соглашением http://www . mathnet.ru/rus/agreement

Параметры загрузки:

IP : 54.197 .217 .227

26 апреля 2023 г., 17:02:43

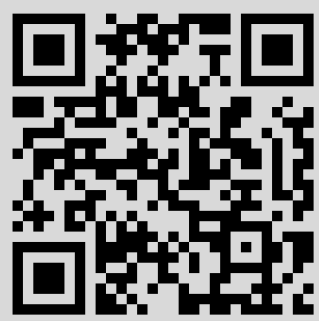




\title{
О ПРИМЕНЕНИИ УСРЕДНЕНИЯ КРЫЛОВА-БОГОЛЮБОВА-МИТРОПОЛЬСКОГО К ПОСТРОЕНИЮ ЭФФЕКТИВНЫХ ГАМИЛЬТОНИАНОВ В ТЕОРИИ СИЛЬНО КОРРЕЛИРОВАННЫХ ЭЛЕКТРОННЫХ СИСТЕМ
}

\begin{abstract}
Показано, что усреднение Крылова-Боголюбова-Митропольского в канонической формулировке может быть использовано как метод построения эффективных гамильтонианов в теории сильно коррелированных электронных систем. В качестве примера рассмотрен переход от гамильтонианов моделей Хаббарда и Андерсона к гамильтонианам $t$ - $J$-модели и модели Кондо соответственно. Этот метод имеет большую степень общности, а также ряд преимуществ перед другими, и его можно применять к решению широкого круга задач физики коррелированных систем.
\end{abstract}

Ключевые слова: усреднение Крылова-Боголюбова-Митропольского, эффективный гамильтониан, модель Хаббарда, $t$ - $J$-модель, модель Андерсона, модель Кондо.

Модельные гамильтонианы, используемые для описания сильно коррелированных электронных систем, в которых потенциальная энергия электронов много больше кинетической, могут быть в значительной мере упрощены путем сведения их к эффективным гамильтонианам в спиновых переменных. Такое упрощение достигается ренормировкой исходного гамильтониана при исключении высокоэнергетических состояний и переходе в подпространство с более низкими энергиями квантовых состояний. Устранение высокоэнергетических состояний оправдано, так как в обычных лабораторных условиях свойства системы, например электропроводность, намагниченность и т.д., определяются основным состоянием и низкоэнергетическими возбуждениями. Для построения эффективных гамильтонианов, как правило, используется метод канонического преобразования [1], [2], с помощью которого добиваются исключения в исходном гамильтониане "недиагонального" оператора малого возмущения, вызывающего переходы между состояниями с низкой и высокой энергиями. Иногда также применяется теория возмущений для систем с вырожденными состояниями [3].

*Научно-практический центр НАН Беларуси по материаловедению, Минск, Беларусь. E-mail: saiko@ifttp.bas-net.by 
В настоящей работе предложен способ построения эффективных гамильтонианов, который основан на использовании метода усреднения Крылова-Боголюбова-Митропольского (KБM) [4], разработанного для решения задач в области теории нелинейных колебаний. Метод усреднения позволяет избавляться в любом порядке по возмущению от быстроосциллирующих членов (высокоэнергетических состояний) в гамильтониане, записанном в представлении взаимодействия, что в результате приводит к эффективному гамильтониану в приближенно-“диагональной” форме.

Ниже для примера мы покажем, как с помощью метода усреднения КБМ в его канонической формулировке из гамильтониана Хаббарда можно получить гамильтониан $t$ - $J$-модели, а из гамильтониана модели Андерсона - гамильтониан модели Кондо.

В теории сильно коррелированных электронных систем парадигматической моделью является модель Хаббарда. Гамильтониан Хаббарда записывается в виде [1], [2]

$$
H=-t \sum_{\langle i j\rangle, \sigma}\left(c_{i \sigma}^{+} c_{j \sigma}+\text { э.c. }\right)+U \sum_{i} n_{i \uparrow} n_{i \downarrow} \equiv H_{t}+H_{U},
$$

где $H_{t}$ - кинетический (зонный) член, описывающий движение по узлам решетки, $H_{U}$ - оператор кулоновской энергии отталкивания двух электронов на одном узле, $c_{i \sigma}^{+}$- оператор рождения электрона со спином $\sigma$ на узле $i, t$ - матричный элемент перехода электрона с данного узла на соседний. При большой кулоновской энергии $U$ появление двух электронов на одном узле становится энергетически невыгодным, и исходная зона расщепляется на две подзоны Хаббарда: верхнюю и нижнюю (с щелью между ними), соответствующие одноэлектронным и двухэлектронным состояниям. В случае половинного заполнения зоны (один электрон на узел, $\left.n=\sum_{\sigma} n_{\sigma}=1\right)$ при $U \sim t$ происходит моттовский переход, т.е. возникает диэлектрическое основное состояние, а между электронами на узлах устанавливается косвенная обменная связь антиферромагнитного типа. Таким образом, реализуется так называемая $t$ - $J$-модель, которая при $n<1$ описывает распространение дырок в нижней подзоне на фоне взаимодействующих спинов. При выводе $t$ - $J$-модели из модели Хаббарда в качестве нулевого приближения берется кулоновский член, а кинетический член рассматривается в качестве возмущения. При переходе к представлению взаимодействия, когда в качестве нулевого гамильтониана выбирается $H_{U}$, кинетический член разбивается на части, описывающие энергетически более выгодные процессы (происходящие внутри подзон Хаббарда) и менее выгодные (происходящие между подзонами Хаббарда). Действительно, в этом представлении

$$
c_{j \sigma}(\tau)=e^{i H_{U} \tau} c_{j \sigma} e^{-i H_{U} \tau}=c_{j \sigma}\left(1-n_{j \bar{\sigma}}\right)+e^{-i U \tau} c_{j \sigma} n_{j \bar{\sigma}},
$$

где $\bar{\sigma}=-\sigma$, а кинетический член $H_{t}(\tau)=e^{i H_{U} \tau} H_{t} e^{-i H_{U} \tau}$ приобретает явно выраженный многочастичный характер:

$$
\begin{aligned}
H_{t}(\tau)= & -t \sum_{\langle i j\rangle, \sigma}\left[\left(1-n_{i \bar{\sigma}}\right) c_{i \sigma}^{+} c_{j \sigma}\left(1-n_{j \bar{\sigma}}\right)+n_{i \bar{\sigma}} c_{i \sigma}^{+} c_{j \sigma} n_{j \bar{\sigma}}+\text { э.c. }\right]- \\
& -t \sum_{\langle i j\rangle, \sigma}\left[n_{i \bar{\sigma}} c_{i \sigma}^{+} c_{j \sigma}\left(1-n_{j \bar{\sigma}}\right)+n_{j \bar{\sigma}} c_{j \sigma}^{+} c_{i \sigma}\left(1-n_{i \bar{\sigma}}\right)\right] e^{i U \tau}-
\end{aligned}
$$




$$
\begin{aligned}
& -t \sum_{\langle i j\rangle, \sigma}\left[\left(1-n_{i \bar{\sigma}}\right) c_{i \sigma}^{+} c_{j \sigma} n_{j \bar{\sigma}}+\left(1-n_{j \bar{\sigma}}\right) c_{j \sigma}^{+} c_{i \sigma} n_{i \bar{\sigma}}\right] e^{-i U \tau} \equiv \\
\equiv & H_{t}^{0}+H_{t}^{+}(\tau)+H_{t}^{-}(\tau) .
\end{aligned}
$$

Первый и второй члены в $H_{t}^{0}$ отвечают за кинетику электронов в нижней и верхней подзонах Хаббарда соответственно; $H_{t}^{+}(\tau)$ описывает появление второго электрона на узле, на котором один электрон уже присутствует, т.е. переход из низкоэнергетического состояния в высокоэнергетическое (соответственно из нижней подзоны Хаббарда в верхнюю); $H_{t}^{-}(\tau)$ представляет собой обратный процесс, вследствие которого число дважды оккупированных узлов (узлов с парой электронов) уменьшается на единицу. Наличие быстроосцилирующих множителей $e^{ \pm i U \tau}$ в $H_{t}^{+}(\tau)$ и $H_{t}^{-}(\tau)$ дополнительно указывает на маловыгодность реализации процессов с переходом между подзонами Хаббарда.

K гамильтониану (2) можно применить метод усреднения КБМ, позволяющий исключить быстроосциллирующие "недиагональные" члены $H_{t}^{ \pm}(\tau)$ в любом порядке теории возмущений по $t$ (точнее, по $t / U$ ). Дадим краткое описание метода в каноническом формализме [4]-[6].

Рассмотрим уравнение Лиувилля для матрицы плотности квантовой системы

$$
\frac{\partial \rho}{\partial \tau}=-i\left[H_{0}+H_{1}, \rho\right] \equiv-i\left(L_{0}+L_{1}\right) \rho,
$$

где $H_{0}$ - невозмущенный “диагональный” гамильтониан системы, $H_{1}$ - малое возмущение - "недиагональный член”, $L_{0}$ и $L_{1}$ - соответствующие гамильтонианам лиувиллианы. В силу условия $\left\|H_{0}\right\| \gg\left\|H_{1}\right\|$, где $\|$. . $\|$ означает величину оператора в единицах частоты, быстрое движение с периодом $2 \pi /\left\|H_{0}\right\|$ налагается на более медленный процесс, характеризующийся временем порядка $\left\|H_{1}\right\|^{-1}$. В уравнении (3) можно перейти к представлению взаимодействия $\sigma=e^{i L_{0} t} \rho, L_{1}(\tau)=e^{i L_{0} \tau} L_{1} e^{-i L_{0} \tau}$ и к уравнению

$$
\frac{\partial \sigma}{\partial \tau}=-i L_{1}(\tau) \sigma
$$

применить метод усреднения КБМ, чтобы исключить быстроосциллирующие члены и построить приближенно-“диагональный” эффективный гамильтониан (лиувиллиан). Кратко опишем эту процедуру в наиболее простом виде.

Отметим, что функция $L_{1}(\tau)$, являясь периодической, может быть разложена в ряд Фурье:

$$
L_{1}(\tau)=\sum_{n} L_{1}^{(n)} e^{i \omega_{n} \tau}, \quad L_{1}^{(n)}=\frac{1}{T} \int_{0}^{T} d \tau L_{1}(\tau) e^{-i \omega_{n} \tau},
$$

где $\omega_{n}=2 \pi n / T, T$ - период, который может, в частности, совпадать с периодом $2 \pi /\left\|H_{0}\right\|$ или быть ему кратным. Поскольку мы интересуемся не быстрым временнь́м "дрожанием" матрицы плотности, а ее медленной эволюцией, т.е. движением, усредненным по нескольким временны́м периодам $T$, то естественно определить операцию усреднения

$$
P^{\tau} \sigma(\tau)=\frac{1}{T} \int_{0}^{T} d \tau \sigma(\tau) \equiv\langle\sigma(\tau)\rangle
$$


где проекционный оператор $P^{\tau}$ осуществляет усреднение быстро изменяющихся величин. Определим также $Q^{\tau}=1-P^{\tau}$ (нетрудно видеть, что $P^{\tau} P^{\tau}=P^{\tau}$, $\left.Q^{\tau} Q^{\tau}=Q^{\tau}, P^{\tau} Q^{\tau}=Q^{\tau} P^{\tau}=0\right)$. Тогда можно записать

$$
\sigma(\tau)=P^{\tau} \sigma(\tau)+Q^{\tau} \sigma(\tau)
$$

т.е. мы разлагаем действительное движение системы, описываемое матрицей плотности $\sigma(\tau)$, на усредненное $P^{\tau} \sigma$ и быстрое “дрожание" $Q^{\tau} \sigma$. Подставляя выражение (7) в уравнение (4) и действуя на него слева операторами $P^{\tau}$ и $Q^{\tau}$ поочередно, получаем два связанных уравнения:

$$
\begin{aligned}
\frac{\partial}{\partial \tau}\left(P^{\tau} \sigma\right) & =-i P^{\tau} L_{1}(\tau) P^{\tau} \sigma-i P^{\tau} L_{1}(\tau) Q^{\tau} \sigma \\
\frac{\partial}{\partial \tau}\left(Q^{\tau} \sigma\right) & =-i Q^{\tau} L_{1}(\tau) Q^{\tau} \sigma-i Q^{\tau} L_{1}(\tau) P^{\tau} \sigma .
\end{aligned}
$$

При выводе уравнений (8), (9) мы воспользовались свойством периодичности функции $Q^{\tau} \sigma$ и тем, что $P^{\tau} \sigma$ - медленная функция времени. Решение уравнения (9) формально можно представить в виде

$$
Q^{\tau} \sigma=-i \int^{\tau} d \tau^{\prime} Q^{\tau^{\prime}} L_{1}\left(\tau^{\prime}\right) P^{\tau^{\prime}} \sigma\left(\tau^{\prime}\right)-i \int^{\tau} d \tau^{\prime} Q^{\tau^{\prime}} L_{1}\left(\tau^{\prime}\right) Q^{\tau^{\prime}} \sigma\left(\tau^{\prime}\right),
$$

причем операторная постоянная в данном выражении положена равной нулю: мы воспользовались правом произвола в выборе этой константы, так как на предыдущем этапе одно дифференциальное уравнение первого порядка (4) было расщеплено на два: (8) и (9). Итерируя выражение (10), получаем разложение по степеням $L_{1}$ :

$$
Q^{\tau} \sigma=\left(-i \int^{\tau} d \tau^{\prime} Q^{\tau^{\prime}} L_{1}\left(\tau^{\prime}\right)-i \int^{\tau} d \tau^{\prime} \int^{\tau^{\prime}} d \tau^{\prime \prime} Q^{\tau^{\prime}} L_{1}\left(\tau^{\prime}\right) Q^{\tau^{\prime \prime}} L_{1}\left(\tau^{\prime \prime}\right) \ldots\right)\langle\sigma\rangle,
$$

где медленно изменяющаяся функция $P^{\tau} \sigma \equiv\langle\sigma\rangle$ вынесена из-под знака интегрирования. Подставляя формулу (11) в уравнение (8), получаем уравнение для медленно изменяющихся величин. Например, во втором порядке по $L_{1}$ имеем

$$
\frac{\partial}{\partial \tau}\langle\sigma\rangle=-i\left\langle L_{1}(\tau)\right\rangle\langle\sigma\rangle-\left\langle\int^{\tau} d \tau^{\prime}\left\{L_{1}\left(\tau^{\prime}\right)\left(L_{1}\left(\tau^{\prime}\right)-\left\langle L_{1}\left(\tau^{\prime}\right)\right\rangle\right)\right\}\right\rangle\langle\sigma\rangle \equiv-i L_{\text {eff }}\langle\sigma\rangle,
$$

где введен эффективный лиувиллиан

$$
L_{\mathrm{eff}}=\left\langle L_{1}(\tau)\right\rangle-i\left\langle\int^{\tau} d \tau^{\prime}\left\{L_{1}\left(\tau^{\prime}\right)\left(L_{1}\left(\tau^{\prime}\right)-\left\langle L_{1}\left(\tau^{\prime}\right)\right\rangle\right)\right\}\right\rangle
$$

которому соответствует эффективный гамильтониан

$$
H_{\mathrm{eff}}=\left\langle H_{1}(\tau)\right\rangle+\frac{i}{2}\left\langle\left[\int^{\tau} d \tau^{\prime}\left(H_{1}\left(\tau^{\prime}\right)-\left\langle H_{1}\left(\tau^{\prime}\right)\right\rangle\right), H_{1}(\tau)\right]\right\rangle .
$$

Для вывода выражения (14) из (13) удобнее всего воспользоваться разложением Фурье (5). 
В модели Хаббарда, рассматриваемой в настоящей работе, усреднение по периоду $2 \pi / U$ гамильтониана $H_{t}(\tau)$ дает $\left\langle H_{t}(\tau)\right\rangle=H_{t}^{0}$, так как средние от $H_{t}^{ \pm}(\tau)$ благодаря множителям $e^{ \pm i U \tau}$ равны нулю. Применение процедуры усреднения КБМ в полном объеме, т.е. использование формулы (14), дает

$$
\begin{aligned}
H_{\mathrm{eff}}^{(2)} & =H_{t}^{0}+\frac{1}{U}\left\langle\left[H_{t}^{+}(\tau), H_{t}^{-}(\tau)\right]\right\rangle+\frac{1}{2 U}\left\langle\left[H_{t}^{+}(\tau)-H_{t}^{-}(\tau), H_{t}^{0}\right]\right\rangle= \\
& =H_{t}^{0}+\frac{1}{U}\left[H_{t}^{+}(0), H_{t}^{-}(0)\right] .
\end{aligned}
$$

Следует отметить, что метод канонического преобразования для гамильтониана Хаббарда приводит к похожему эффективному гамильтониану, но с дополнительными "недиагональными" членами $\sim\left[H_{t}^{ \pm}(0), H_{t}^{0}\right]$ (см., например, работы [1], [2]), которыми можно пренебречь на том основании, что они учитывают межзонные переходы только во втором порядке по $t / U$ [2], а не в первом. В методе КБМ такие члены не возникают, так как при усреднении по периоду $2 \pi / U$ быстроосциллирующий член $\left[H_{t}^{+}(\tau)-H_{t}^{-}(\tau), H_{t}^{0}\right]$ в $(15)$ обращается в нуль. Далее, чтобы получить искомый результат, необходимо в выражении (15) подставить $H_{t}^{ \pm}(0)$ из формулы (2) и проделать достаточно простые операции, подробно описанные в работе [1]. А именно: выполнить коммутации операторов, отбросить трехузельные члены [1], [2] и взять проекцию полученного гамильтониана на нижнюю подзону Хаббарда, т.е. отбросить члены, слева и справа умноженные на операторы $n_{i \bar{\sigma}}, n_{j \bar{\sigma}}$, такие, например, как второй член в $H_{t}^{0}(2): n_{i \bar{\sigma}} c_{i \sigma}^{+} c_{j \sigma} n_{j \bar{\sigma}}$ (подобные члены описывают высокоэнергетическое движение электронов в верхней подзоне Хаббарда). Полученный в результате эффективный гамильтониан $H_{\mathrm{eff}}^{(2)}$ есть не что иное, как гамильтониан $t$ - $J$-модели:

$$
H_{\mathrm{eff}}^{(2)} \rightarrow H_{t-J}=-t \sum_{i, j, \sigma}\left[\left(1-n_{i \bar{\sigma}}\right) c_{i \sigma}^{+} c_{j \sigma}\left(1-n_{j \bar{\sigma}}\right)+\text { э.c. }\right]+J \sum_{i, j}\left(\vec{S}_{i} \vec{S}_{j}-\frac{1}{4} n_{i} n_{j}\right)
$$

где

$$
\vec{S}_{i}=\frac{1}{2} \sum_{\sigma \sigma^{\prime}} c_{i \sigma}^{+} \vec{\tau}_{\sigma \sigma^{\prime}} c_{i \sigma^{\prime}}
$$

$\vec{\tau}$ - вектор, составленный из матриц Паули, $n_{i}=\sum_{\sigma} n_{i \sigma}, J=4 t^{2} / U$.

Описанный выше алгоритм построения эффективного гамильтониана применим и при преобразовании гамильтониана модели Андерсона к гамильтониану модели Кондо. Переход модель Андерсона - модель Кондо обычно осуществляют с помощью преобразования Шриффера-Вольфа [7], [8], т.е. канонического преобразования с антиэрмитовским оператором, форма которого подбирается.

Применение метода усреднения КБМ и в этом случае имеет несомненные преимущества. Исходный гамильтониан Андерсона записывается в виде [7], [8]

$$
\begin{gathered}
H=H_{0}+V \\
H_{0}=\sum_{k, \sigma} \varepsilon_{k} c_{k \sigma}^{+} c_{k \sigma}+\varepsilon \sum_{\sigma} c_{d \sigma}^{+} c_{d \sigma}+U c_{d \uparrow}^{+} c_{d \uparrow} c_{d \downarrow}^{+} c_{d \downarrow} \\
V=\sum_{k, \sigma}\left(V_{k d} c_{k \sigma}^{+} c_{d \sigma}+\text { э.c. }\right)
\end{gathered}
$$


где $c_{k \sigma}^{+}, \varepsilon_{k}$ - соответственно оператор рождения и энергия электрона в зоне проводимости с импульсом $k$ и спином $\sigma ; c_{d \sigma}^{+}$и $\varepsilon$ - соответственно оператор рождения и энергия локализованного электрона примесного атома, $U$ - кулоновская энергия взаимодействия между двумя электронами, оккупирующими примесный атом; $V$ описывает взаимодействие, смешивающее примесные состояния с зонными, а $V_{k d}-$ константа такого взаимодействия.

Для перехода к представлению взаимодействия $V(\tau)=e^{i H_{0} \tau} V e^{-i H_{0} \tau}$ запишем сначала $c_{k \sigma}(\tau)=e^{i H_{0} \tau} c_{k \sigma} e^{-i H_{0} \tau}$ и $c_{d \sigma}(\tau)=e^{i H_{0} \tau} c_{d \sigma} e^{-i H_{0} \tau}$ в виде

$$
c_{k \sigma}(\tau)=c_{k \sigma} e^{-i \varepsilon_{k} \tau}, \quad c_{d \sigma}(\tau)=c_{d \sigma}\left(1-n_{d \bar{\sigma}}\right) e^{-i \varepsilon \tau}+c_{d \sigma} n_{d \bar{\sigma}} e^{-i(\varepsilon+U) \tau} .
$$

В результате получаем

$$
V(\tau)=\sum_{k, \sigma}\left\{V_{k d}\left[e^{i\left(\varepsilon_{k}-\varepsilon\right) \tau} c_{k \sigma}^{+} c_{d \sigma}\left(1-n_{d \bar{\sigma}}\right)+e^{i\left(\varepsilon_{k}-\varepsilon-U\right) \tau} c_{k \sigma}^{+} c_{d \sigma} n_{d \bar{\sigma}}\right]+\text { э.c. }\right\} .
$$

Пусть все энергии отсчитываются от уровня Ферми, тогда в интересующей нас области температур энергии $\varepsilon_{k}$ (или $\varepsilon_{k}-\mu$ вместо $\varepsilon_{k}$, где $\mu$ - энергия Ферми) малы, $\varepsilon$ - отрицательно, причем $U,|\varepsilon| \gg \varepsilon_{k}$.

Усреднение по времени гамильтониана $V(\tau)$ дает нуль в первом порядке возмущения из-за быстрых осцилляций множителей $e^{ \pm i\left(\varepsilon_{k}-\varepsilon\right) \tau}, e^{ \pm i\left(\varepsilon_{k}-\varepsilon-U\right) \tau}$. Во втором порядке усреднение КБМ (14) приводит к следующему результату:

$$
\begin{aligned}
H_{\mathrm{eff}}^{(2)}(\tau)= & \frac{i}{2}\left\langle\left[\int^{\tau} d \tau^{\prime} V\left(\tau^{\prime}\right), V(\tau)\right]\right\rangle=-\frac{1}{2} \sum_{k, k^{\prime}, \sigma}\left[f\left(k, k^{\prime}, U\right)-f\left(k, k^{\prime}, 0\right)\right] \times \\
& \times\left(c_{k \sigma}^{+} c_{k^{\prime} \sigma} c_{d \bar{\sigma}}^{+} c_{d \sigma}-c_{k \sigma}^{+} c_{k^{\prime} \sigma} c_{d \bar{\sigma}}^{+} c_{d \bar{\sigma}}\right) e^{i\left(\varepsilon_{k}-\varepsilon_{k^{\prime}}\right) \tau}+ \\
& +\frac{1}{2} \sum_{k, k^{\prime}, \sigma} f\left(k, k^{\prime}, 0\right) c_{k \sigma}^{+} c_{k^{\prime} \sigma} e^{i\left(\varepsilon_{k}-\varepsilon_{k^{\prime}}\right) \tau}- \\
& -\frac{1}{2} \sum_{k, \sigma} f(k, k, 0) n_{d \sigma}-\frac{1}{2} \sum_{k, \sigma}[f(k, k, U)-f(k, k, 0)] n_{d \sigma} n_{d \bar{\sigma}},
\end{aligned}
$$

где

$$
f\left(k, k^{\prime}, U\right)=V_{k d} V_{k^{\prime} d}^{*}\left(\frac{1}{\varepsilon_{k}-\varepsilon-U}+\frac{1}{\varepsilon_{k^{\prime}}-\varepsilon-U}\right),
$$

$e^{i\left(\varepsilon_{k}-\varepsilon_{k^{\prime}}\right) \tau}-$ медленно изменяющиеся множители. Переходя в выражении $(22)$ к представлению Гейзенберга, получаем эффективный гамильтониан

$$
H_{\mathrm{eff}}^{(2)}(\tau) \rightarrow H_{\mathrm{eff}, H}^{(2)}=H_{0}+H_{\mathrm{eff}}^{(2)}(0),
$$

где $H_{0}$ определено формулой (18). При использовании преобразования ШриффераВольфа в гамильтониане (23) появляются дополнительные члены вида [7], [8]

$$
H_{\delta}=-\frac{1}{2} \sum_{k, k^{\prime}, \sigma} \frac{V_{k^{\prime} d}}{V_{k^{\prime} d}^{*}}\left[f\left(k, k^{\prime}, U\right)-f\left(k, k^{\prime}, 0\right)\right]\left(c_{k \sigma} c_{k^{\prime} \bar{\sigma}} d_{\bar{\sigma}}^{+} d_{\sigma}^{+}+\text {э.c. }\right),
$$


описывающие высокоэнергетические процессы: изменение населенности примесного уровня за счет захвата двух зонных электронов или перехода двух электронов с $d$-орбитали в зону проводимости. Такие члены при применении метода КБМ отсутствуют в эффективном гамильтониане, так как в представлении взаимодействия они являются быстроосциллирующими и поэтому зануляются при выполнении процедуры усреднения. Пренебрегая в $H_{\mathrm{eff}}^{(2)}(0)$ членами, описывающими потенциальное рассеяние электронов проводимости (второй член) и перенормировку энергии электронов на примесном атоме (третий и четвертый члены), а также кулоновский (высокоэнергетический) член в $H_{0}$, из уравнения (23) с учетом выражений $(18),(22)$ получаем гамильтониан модели Кондо:

$$
\begin{aligned}
H_{\mathrm{eff}, H}^{(2)} \rightarrow H_{\text {Kondo }} & =\sum_{k, \sigma} \varepsilon_{k} c_{k \sigma}^{+} c_{k \sigma}- \\
& -\frac{1}{2} \sum_{k, k^{\prime}, \sigma}\left[f\left(k, k^{\prime}, U\right)-f\left(k, k^{\prime}, 0\right)\right]\left(c_{k \sigma}^{+} c_{k^{\prime} \bar{\sigma}} c_{d \bar{\sigma}}^{+} c_{d \sigma}-c_{k \sigma}^{+} c_{k^{\prime} \sigma} c_{d \bar{\sigma}}^{+} c_{d \bar{\sigma}}\right),
\end{aligned}
$$

в котором член взаимодействия может быть выражен через спиновые переменные зонных электронов и электронов на $d$-орбитали примесного атома [7], [8]. С помощью метода усреднения КБМ можно рассматривать и системы, находящиеся в неравновесных условиях; например, его можно применять при изучении эффекта Кондо в случае, если энергия электрона, локализованного на квантовой точке, модулируется внешним переменным электрическим полем.

Таким образом, усреднение КБМ является надежно работающим методом при построении эффективных гамильтонианов для сильно коррелированных электронных систем: высокотемпературных сверхпроводников, оксидных магнетиков с колоссальным магнитосопротивлением, квантовых точек и т.д. Применение этого метода показано на примере преобразования гамильтониана Хаббарда к гамильтониану $t$ - $J$ модели, гамильтониана Андерсона к гамильтониану модели Кондо. Алгоритм вычислений оказывается достаточно простым и естественным. При применении метода усреднения не требуется той изобретательности, которая нужна при выборе конкретного вида унитарного оператора в случае использования канонического преобразования. Также нет необходимости знать собственные функции и собственные значения гамильтониана нулевого приближения, как в случае применения теории возмущений для систем с вырожденными состояниями. Кроме того, новый метод самодостаточен: он не приводит к появлению "недиагональных" членов высших порядков по параметру возмущения в преобразованном гамильтониане, которые, как правило, возникают при использовании канонического преобразования и затем отбрасываются на основе правдоподобных соображений. Этот подход обладает большой степенью общности и может быть применим к решению широкого круга задач физики сильно коррелированных электронных систем. В частности, его можно применить к выводу эффективного гамильтониана (типа $t$ - $J$-модели) для недавно открытого класса высокотемпературных сверхпроводников, представляющих собой слоистые соединения на основе железа (см. работу [9]). 


\section{Список литературы}

[1] P. Fazekas, Lecture Notes on Electron Correlation and Magnetism, World Sci., Singapore, 1999.

[2] Ю. А. Изюмов, УФН, 167:5 (1997), 465-497.

[3] C. L. Cleveland, R. Medina, Amer. J. Phys., 44:1 (1976), 44-46.

[4] Н.Н. Боголюбов, Ю.А. Митропольский, Асимптотические методы в теории нелинейных колебаний, Наука, М., 1974.

[5] Л. Л. Буишвили, М. Г. Менабде, ЖЭТФ, 77:6(12) (1979), 2435-2442.

[6] А. П. Сайко, ФTT, 35 (1993), 38.

[7] J. R. Schrieffer, P. A. Wolff, Phys. Rev., 149:2 (1966), 491-492.

[8] P. Phillips, Advanced Solid State Physics, Westview Press, Boulder, 2003.

[9] Ю. А. Изюмов, Э. З. Курмаев, УФН, 178:12 (2008), 1307-1334.

Поступила в редакцию 19.12.2006, после доработки 12.03.2009 